\title{
Manifestaciones cardiovasculares asociadas a enfermedad por COVID - 19
}

\section{Cardiovascular manifestations associated with COVID-19 disease}

Recibido: $18 / 06 / 2021$ Aceptado: 22/07/2021
Ricardo Andrés García Archila

Universidad San Carlos de Guatemala ricardoandres3007@gmail.com

https://orcid.org/0000-0002-8251-9070

\section{Referencia del artículo}

García Archila , R. A. (2021). Manifestaciones cardiovasculares asociadas a enfermedad por COVID - 19. Revista Diversidad Científica, 1(1), 9-14. DOl: https://doi.org/10.36314/diversidad.v1i1.2

\section{Resumen}

EI COVID-19 es una infección viral producida por el SARS-CoV-2, afectando principalmente las vías respiratorias, sin embargo, se han estudiado otros órganos afectados, entre ellos el sistema cardiovascular. OBJETIVO: describir las principales manifestaciones cardiovasculares en los pacientes con enfermedad COVID-19 e identificar los factores de riesgo relacionados con mayor frecuencia a complicaciones cardiovasculares en enfermedad por COVID-19. MÉTODO: se realizó una revisión sistemática de distintas fuentes médicas incluyendo la información más actualizada. RESULTADOS: las principales manifestaciones cardiacas en pacientes con COVID-19 encontradas fueron miocarditis, insuficiencia cardiaca descompensada, síndrome coronario agudo, arritmias y eventos tromboembólicos, además, los factores de riesgo relacionados con mayor frecuencia a complicación cardiovascular en COVID-19 fueron el sexo masculino, edad avanzada y enfermedad cardiovascular preexistente, como hipertensión arterial e insuficiencia cardiaca. CONCLUSIÓN: las manifestaciones cardiacas fueron evidentes, teniendo una repercusión desfavorable en el pronóstico, por lo que una evaluación cardiológica es fundamental en todo paciente con COVID-19.

Palabras clave: COVID-19, arritmias, infección viral, cardiovascular 


\begin{abstract}
COVID-19 is a viral infection produced by SARS-CoV-2, mainly affecting the respiratory tract, however, other affected organs have been studied, including cardiology. OBJECTIVE: to describe the main cardiovascular manifestations in patients with COVID-19 disease and to identify the risk factors most frequently related to cardiovascular complications in COVID-19 disease. METHOD: a systematic review of different medical sources including the most up-to-date information was carried out. RESULTS: the main cardiac manifestations found in patients with COVID-19 were myocarditis, decompensated heart failure, acute coronary syndrome, arrhythmias and thromboembolic events, in addition, the risk factors most frequently related to cardiovascular complications in COVID-19 were male, advanced age, and pre-existing cardiovascular disease, such as high blood pressure and heart failure. CONCLUSION: the cardiac manifestations were evident, having an unfavorable impact on the prognosis, so a cardiological evaluation is essential in all patients with COVID-19.
\end{abstract}

Keywords: COVID-19, arrhythmias, viral infection, cardiovascular 


\section{Introducción}

En diciembre de 2019 se identificó en Wuhan una serie de casos de neumonía originados por un nuevo coronavirus al cual denominaron, según la organización mundial de la salud, SARS-Cov-2 (OPS \& OMS, 2020).

A pesar de que el cuadro clínico principal de COVID-19 se ha descrito como respiratorio, deben reconocerse las demás características y otras manifestaciones asociadas a otros aparatos y sistemas, como las cardiovasculares (Clemente, Sanchez, \& Enríquez, 2020).

En los primeros 72,314 casos de COVID-19 se pudo observar que el $81 \%$ presentaron infección leve, con una mortalidad de $2.3 \%$, mientras que en los pacientes que se consideraron críticos se documentó una mortalidad del $50 \%$. En otra cohorte de Wuhan, la necesidad de ser ingresado en una unidad de cuidado intensivo fue del $26 \%$ de estos, el $60 \%$ fue por síndrome de dificultad respiratoria aguda, mientras que el $40 \%$ fue por arritmias cardiacas (Palacios, Santos, Velázquez, \& León, 2021).

El objetivo del presente artículo es describir las principales manifestaciones cardiovasculares en los pacientes con enfermedad COVID-19, así mismo identificar los factores de riesgo relacionados con mayor frecuencia a complicaciones cardiovasculares en enfermedad por COVID-19, ya que estos datos constituyen una piedra angular en la evaluación y manejo integral del paceinte con enfermedad por COVID-19.

\section{Contenido}

La edad avanzada, sexo masculino y enfermedad cardiovascular preexistente como hipertensión arterial e insuficiencia cardíaca son factores de riesgo importantes que se relacionan a enfermedad grave por COVID-19.

Según Valenzuela y Amado, en el estudio "Compromiso cardiovascular en COVID19 " en el cual se llevó a cabo un meta-análisis de seis estudios con 1527 pacientes incluidos, y en el que se estudiaron algunos factores de riesgo y su relación con enfermedad grave por COVID-19, se determina que en casos graves, las incidencias de hipertensión, enfermedad cardiovascular y cerebrovascular y diabetes se duplicaron y triplicaron, o duplicaron en los casos admitidos en $\mathrm{UCl}$, en comparación con aquellos que no cumplieron dicha condición. Así mismo, un $18 \%$ de los pa- 
cientes presentó injuria cardíaca aguda, una cifra 13 veces más alta en casos admitidos en $\mathrm{UCl}$ en comparación con los no admitidos. Dichos datos coinciden con el presente estudio, indicando que la presencia de antecedentes cardiovasculares en los pacientes con COVID-19 aumenta drásticamente el riesgo de sufrir enfermedad grave y necesitar atención en Unidad de Cuidados Intensivos (Valenzuela \& Amado, 2019).

En este estudio se concluye que la miocarditis, insuficiencia cardiaca descompensada, síndrome coronario agudo y eventos tromboembólicos son manifestaciones clínicas importantes a nivel cardiovascular en COVID-19.

En un estudio de 150 pacientes, realizado por Ricaurte et al, se documentó miocarditis secundaria a inflamación daño al miocardio en COVID-19; en dicho estudio se presentaron 68 muertes, de las cuales el $7 \%$ se relaciona a miocarditis con insuficiencia circulatoria y en un $33 \%$ de los casos la miocarditis desempeñó posiblemente un papel importante en la muerte del paciente. Así mismo, se describe la existencia de miocarditis fulminante en presencia de carga viral alta, en autopsia realizadas en dichos cadáveres se evidenció un infiltrado inflamatorio mononuclear en el tejido miocárdico. Estos datos resaltan la importancia de un abordaje integral en pacientes con infección por COVID-19, incluyendo una evaluación cardiológica completa (Ricaurte, Hernandez, \& Garnica, 2020).

\section{Conclusión}

Las manifestaciones cardiovasculares identificadas con mayor frecuencia en pacientes con COVID-19 incluyen miocarditis, insuficiencia cardiaca descompensada, síndrome coronario agudo y eventos tromboembólicos, no debiendo pasarse estas desapercibidas en la evaluación del paciente (Noria, Bachini, \& Ramos, 2020).

Dentro de los factores de riesgo relacionados con mayor frecuencia a complicaciones cardiovasculares en infección por COVID-19 se identificaron el sexo masculino, edad avanzada y presencia de enfermedad cardiovascular preexistente como la hipertensión arterial, enfermedad cerebrovascular y diabetes mellitus, así mismo estas patologías se asocian a pronóstico desfavorable (Clemente, Sanchez, \& Enríquez, 2020). 


\section{Revista Diversidad Científica Vol. 1 No. 1 Año 2021}

\section{Referencias}

Clemente, A., Sanchez, E., \& Enríquez, M. (2020). Manifestaciones cardiológicas en pacientes con COVID 19. Revista de Medicina Interna de México, 357-364. https://doi.org/10.24245/mim.v36i3.4229

Noria, S., Bachini, J. P., \& Ramos, M. V. (2020). Coronavirus y sistema cardiovascular. Revista Uruguaya de Cardiología, 221-226. https://doi.org/10.29277/ cardio.35.2.13

OPS, \& OMS. (14 de Febrero de 2020). OPS/OMS. Obtenido de https://www3.paho. org/hq/index.php?option=com_docman\&view=list\&slug=2020-alertas-epidemiologicas\&ltemid=270\&layout=default\&lang=es

Palacios, M., Santos, E., Velázquez, M., \& León, M. (2021). COVID-19, una emergencia de salud pública mundial. Revista Clínica Española, 55-61. https://doi. org/10.1016/j.rce.2020.03.001

Ricaurte, A., Hernandez, V., \& Garnica, M. (2020). Características clínicas de las manifestaciones extrapulmonares de la infección COVID-19. Salutem Scientia Spiritus, 116-123. Obtenido de https://revistas.javerianacali.edu.co/index.php/ salutemscientiaspiritus/article/view/2323/2888

Valenzuela, G., \&Amado, P. (2019). Compromiso cardiovascular en COVID-19. Revista de la Sociedad Peruana de Medicina Interna, 61-67. https://doi.org/10.36393/ spmi.v33i2.522

\section{Sobre el autor}

\section{Ricardo Andrés García Archila}

Es egresado de la carrera Médico y Cirujano de la Universidad de San Carlos de Guatemala. Participó en los proyectos de investigación "Manejo del pie Diabético", "Hallazgos hematológicos en pacientes ingresados por dengue el servicio de pediatría en el Hospital Regional de Zacapa del primero de enero del 2019 al 31 de diciembre del 2019". 
Copyright (c) Ricardo Andrés García Archila

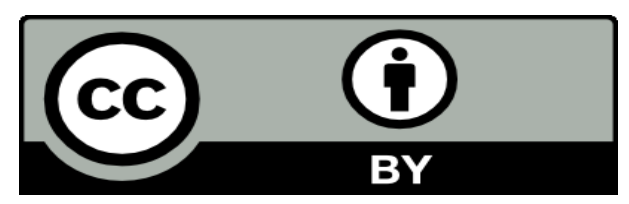

Este texto está protegido por una licencia CreativeCommons 4.0.

Usted es libre para compartir, copiar y redistribuir el material en cualquier medio o formato y adaptar el documento, remezclar, transformar y crear a partir del material para cualquier propósito, incluso comercialmente, siempre que cumpla la condición de atribución: usted debe reconocer el crédito de una obra de manera adecuada, proporcionar un enlace a la licencia, e indicar si se han realizado cambios. Puede hacerlo en cualquier forma razonable, pero no de forma tal que sugiera que tiene el apoyo del licenciante o lo recibe por el uso que hace. 